\title{
PULMONARY HYPERSENSITIVITY DISEASE DUE TO INHALED ORGANIC ANTIGENS
}

\author{
J. PePYS, M.R.C.P., F.R.C.P.E. \\ M.R.C. Clinical Immunology Research Group \\ Institute of Diseases of the Chest, Brompton, London, S.W.3
}

THE MeChanisms of industrial pulmonary disease due to the inhalation of organic dusts are not well understood. Some light may be thrown on these problems by the findings in pulmonary aspergillosis and farmer's lung, which provide comprehensive examples of hypersensitivity diseases of the lungs due to inhaled organic antigens. These somewhat unfamiliar diseases and their various forms are not always clearly distinguished from each other. They encompass, between them, reactions occurring in the bronchi, in and around the bronchi, and in the peripheral gas-exchanging tissues of the lungs, usually called, for lack of a more precise term, the interstitial tissues. These reactions in different parts of the respiratory tract have their own particular clinical and other features, and it is now becoming possible to explain their probable immunopathological mechanisms. Both clinical and immunological findings need to be considered together for diagnostic purposes.

\section{Pulmonary Aspergillosis}

Aspergillus fumigatus is the most important cause of pulmonary aspergillosis, other species of Aspergillus being responsible on rare occasions. The fungus is ubiquitous, and, in Britain, its spores tend to be most prevalent in the air in the winter months, a point of clinical importance. $A$. fumigatus is pathogenic to birds and cattle, and is potentially pathogenic to man. It may act in man as an allergen or antigen, and as a saprophyte or a pathogen, being responsible for different pulmonary reactions consisting of asthma; asthma complicated by pulmonary eosinophilia; aspergillus mycetoma or aspergilloma, and, under special conditions, it may invade the tissues and spread to other parts of the body.

\section{Asthma}

The spores of $A$. fumigatus may, like the spores of other fungi and other inhaled allergens, act as an allergen provoking immediate *(Type I) hypersensitivity reactions. This type of reaction is mediated by non-precipitating, skin-sensitising antibody, known as reagin, demonstrable by the production of immediate wealing reactions on skin testing, and immediate bronchial reactions on inhalation testing. In a survey in which there was some selection of patients (Longbottom and Pepys, 1964), positive skin test reactions to prick test with $A$. fumigatus were reported in 38 per cent of patients with uncomplicated asthma. A lower proportion, about 10 to 20 per cent, of unselected patients with extrinsic asthma give positive reactions to $A$. fumigatus.

The bronchial reaction, which consists of oedema of the bronchial mucosa accompanied by infiltration with eosinophil cells, results in ventilatory obstruction and asthma. The symptoms and signs are those of a bronchial reaction, with wheezing, rhonchi and prolonged expiration, the dyspnoea being proportional to the intensity of the physical signs. The injection of extracts of $A$. fumigatus for treatment purposes in these patients is tolerated well and appears to be of value.

\section{Asthma and Pulmonary Eosinophilia}

Asthma may become complicated by pulmonary eosinophilia, that is, by the appearance of transitory pulmonary infiltrations and eosinophilia of the blood. In the United Kingdom there is evidence that this quite common clinical disorder is due in the majority of cases to A. fumigatus, and occasionally to other members of the Aspergillus genus such as $A$. flavus, $A$. nidulans, $A$. niger and $A$. terreus. The great majority of cases of pulmonary eosinophilia in association with asthma seen in this country are due to pulmonary aspergillosis. The usual history is that the patient, almost always an asthmatic subject with evidence of extrinsic sensitivity to other common allergens, develops a febrile illness, often in the winter months, associated with the coughing up of cord-like plugs of sputum and of small tough lumps which may be light brown or brown in colour. On X-ray, pulmonary infiltration of the collapse-consolidation type appear.

*Types of hypersensitivity as described by Gell and Coombs (1963). 
The asthma usually becomes more persistent and severe, and recurrent infiltrations, lasting from days to several weeks, appear in different areas of the lungs. On occasion, quite large bronchi may become obstructed with collapse of extensive areas of lung, and even of entire lobes. A characteristic bronchiectasis may be found at the sites of the infiltrations, consisting of cylindrical dilatations, with normal filling of the bronchi distal to the dilatation.

Prick tests with extracts of $A$. fumigatus give positive reactions in about $90 \%$ of these patients. A negative reaction to a prick test with $A$. fumigatus tends to exclude the diagnosis. It must be emphasised that a positive reaction to a prick test need consist of only a small weal together with erythema and often with itching. Testing of such patients with stronger extracts than are usually used will give larger weals. In addition to the immediate skin test reactions, precipitins against Aspergillus antigens are present in the sera of about two-thirds of these patients**, in contrast to the patients with asthma only, in whom precipitins have been found in $9 \%$. (Longbottom and Pepys, 1964).

In the patients who give an immediate reaction to skin tests, and who have precipitins as well, a second skin test reaction may develop 3 to 4 hours after the test, particularly if it is performed intracutaneously with purified protein fractions of $A$. fumigatus. This second reaction may be extensive, and consists of an itchy, soft, oedematous swelling, which is usually maximal at about 7 to 8 hours, and then begins to resolve. It appears only rarely in asthmatic patients who give immediate skin test reactions but who have not got precipitins, and does not occur in non-allergic subjects who have precipitins but who do not give immediate skin test reactions. The reaction is regarded as a precipitin-mediated reaction of the Arthus-type (Type 3 hypersensitivity), although the presence of reaginic antibody seems also to be necessary for its production. Further studies are needed to resolve this immunological problem. It differs in its macroscopic appearances and speed of development from the delayed tuberculin (Type 4 hypersensitivity) reaction, in which induration and tense oedematous infiltration of the skin starts to make its appearance after about 24 hours, and is maximal at about 48 to 72 hours.

Culture of the sputum for $A$. fumigatus is of limited value in the diagnosis, since the spores are inhaled by the population at large and can, therefore, be present in the sputum of unaffected subjects. Positive cultures are, however, obtained more frequently from patients with asthma (Pepys, Riddell, Citron, Clayton and Short, 1959). In the

**More recent investigations have shown precipitins in almost all of these subjects if the serum is concentrated. patients with asthma and pulmonary eosinophilia as well, the histological examination of the sputum plugs is helpful, since they may contain fungaी mycelium showing that growth is occurring. It is: not uncommon to obtain negative cultures on number of occasions, followed by a series of positive, and often strongly positive, cultures. seems likely that the growth of the fungus in the bronchi provides a source of antigen sufficient th cause a local hypersensitive reaction in the bronchus like the Arthus-type reaction in the skin, and that this is responsible for the bronchiectasis which $\mathbb{R}$ found.

The diagnostic features of this form of pulmonarg aspergillosis are the presence of asthma, transito recurrent pulmonary infiltrations, blood eosinophiliz. (usually about $1000-1500$ per cu. $\mathrm{mm}$ ), and positive immediate wealing reaction to prick tests The diagnosis is supported by positive precipitif tests, by the production of an Arthus-type as well ais immediate skin test reaction, by the presence of mycelium in the plugs, and by repeated positiv cultures of the sputum.

The causes of other cases of pulmonary eosino philia in Britain are not known. Pulmonary infit trations and eosinophilia of the blood may also bo found in the form of polyarteritis nodosa when presents with asthma, the number of eosinophilo in this condition being usually much higher, and the other evidence of pulmonary aspergillosis be absent. The pulmonary infiltrations may ibs sufficiently persistent to suggest pulmonary tubes culosis, or other forms of pneumonia, due tō aspiration or to viral or other infection. In some patients, in whom a diagnosis of bronchiectasi with wheezing due to lung damage may be made review of the problem along the above lines shows that the asthma preceded the bronchial damage and that the patient belongs in the group of asthma an $\overline{\text { A }}$ pulmonary eosinophilia. In some patients, witt areas of collapse in association with the coughing. of mucous plugs, in whom a diagnosis of mucoie impaction may be made, there is evidence of infection and hypersensitivity to $A$. fumigatus.

Injections of extracts of $A$. fumigatus in these subjects, in contrast to the asthmatic subjects wh\& have reagins only, tend to provoke increasingls severe local and focal reactions, and commonly have. to be discontinued. The inhalation of fungistatiov agents such as brilliant green or Pimafucin may be tried, though their efficacy is not established. Pard of the difficulty here is that re-infection occurs particularly during the winter months. Systemie treatment with antifungal agents has not been found effective. Corticosteroid treatment appearo to be effective in controlling the pulmonary infif trations, and its use is indicated where the asthma ip severe and persistent. It is hoped that prevention of 
the pulmonary infiltrations will also prevent the appearance of the bronchiectasis. The amount of sputum decreases rapidly with corticosteroid treatment, thus showing the importance of the hypersensitivity reaction as against that of the fungal infection "per se."

\section{Aspergillus Mycetoma, or Aspergilloma}

$A$. fumigatus, and, on rare occasions, other members of the Aspergillus genus, may grow as saprophytes in the nasal sinuses, in areas of damaged bronchus such as the open-healed cavities of patients with treated tuberculosis, or in lung bullae, and on occasion in the pleural cavity. The aspergilloma is seen on X-ray as a rounded mass in the lungs (fungal-ball), surrounded by an air halo. The symptoms and signs are chiefly those of a spaceoccupying mass, and there may be haemoptysis. Aspergillomata are not infrequently present without symptoms.

The precipitin test for aspergillus precipitins has a high diagnostic value in these patients, being strongly positive in the great majority of cases, and usually becomes rapidly weaker or negative on removal or expectoration of the mycetoma. Since the aspergilloma usually occurs in non-allergic patients, though it is not confined to them, skin test reactions are negative in most cases. Where asthma is present together with the mycetoma, the allergic form of pulmonary aspergillosis may develop, with the appearance, in addition, of transitory pulmonary infiltrations, and eosinophilia of the blood. The culture of the fungus from the sputum will depend upon the patency of the bronchus leading to the mycetoma. In many cases, therefore, sputum cultures are negative. It should be emphasised that an aspergilloma may, on occasion, be present together with other pathological disorders of the lung, such as a carcinoma or even active tuberculosis. The appearance, due to $A$. fumigatus, of fresh and increasing infiltrations on X-ray in patients with healed tuberculosis may be misleading and suggest reactivation and spread of the disease.

Where the aspergilloma is present without symptoms no treatment is indicated, though in other cases surgical removal may be necessary. Care needs to be taken in the allergic form of pulmonary aspergillosis, since surgical intervention is not appropriate, the condition continuing to progress. The inhalation of fungistatic or fungicidal agents such as brilliant green or Pimafucin may be tried, though if this cannot reach the aspergilloma no effect can be expected.

\section{Miscellaneous Lung Diseases}

$A$. fumigatus not infrequently infects areas of damaged lung. In about $8 \%$ of patients with a variety of lung diseases a weak precipitin reaction may be obtained, probably evidence of past or present infection (Longbottom and Pepys, 1964). In some cases this reaction has led to more detailed search and the discovery by tomography of previously unsuspected aspergillomas; whereas in other cases serial observations have shown the development of an aspergilloma with increasingly strong precipitation reactions.

\section{Invasive Aspergillosis}

Only very limited invasion of the tissues by $A$. fumigatus is found in the other forms of pulmonary aspergillosis. In patients with reticulo-endothelial disease, or who have had intensive X-ray irradiation, invasion of the pulmonary and other tissues of the body by $A$. fumigatus may be found at necropsy.

\section{Farmer's Lung}

Farmer's lung is a hypersensitivity disease due to the inhalation of the dust of mouldy hay or other vegetable matter, with symptoms and signs attributable to a reaction in the most peripheral part of the respiratory tract giving rise to a defect in gasexchange. It has now become a registered industrial disease in the United Kingdom. Repeated exposure to mouldy hay leads to increasing hypersensitivity to the dust so that only minimal exposure may suffice to provoke attacks. The clinical features were originally described as appearing some hours, 5 or 6 or more, after the exposure. It is now evident that the disease may just as often develop insidiously, so that the affected subject may not relate the symptoms to the occupational exposure. Chronic lung damage may follow after only one or many attacks.

The symptoms consist of cough, together with dyspnoea which may be very severe and out of proportion to the crepitant rales which are present. There is little sputum and there may be slight haemoptysis. Fever, shivering attacks or rigors, malaise and loss of weight are also present. Pulmonary function tests show a decrease in $\mathrm{CO}$ gas transfer factor, that is, there is an impairment in ventilation-perfusion, accompanied by a decrease in the compliance of the lungs. These findings are in contrast to the bronchial reaction with disturbance of ventilation which occurs in asthma. On X-ray examination a fine miliary infiltration of the lungs may be found. These infiltrations are quite different from the collapse-consolidation infiltrations of the allergic form of pulmonary aspergillosis.

The acute episodes of farmer's lung may be of short duration clearing up overnight or within a few days, though more prolonged and recurrent attacks due to re-exposure may lead to permanent lung damage with increasing dyspnoea and evidence of diffuse fibrosis of the lungs. The tissue reactions consist in some cases of an infiltration in the alveolar 
walls by lymphocytes, plasma cells and neutrophils (Emanuel, Wenzel, Bowerman and Lawton, 1964), and in others the presence of epithelioid cell granulomata (Dickie and Rankin, 1958). Eosinophilia of the blood, sputum and tissues is not present in farmer's lung, whereas it is a feature of asthma and pulmonary aspergillosis of the allergic type.

The dust of mouldy overheated hay responsible for farmer's lung is rich in micro-organisms and in particular in thermophilic actinomycetes which grow well at high temperatures of 40 to $60^{\circ} \mathrm{C}$. These high temperatures are produced by fungal and bacterial growth in hay which is stored with a content of moisture greater than 29 to $34 \%$ water (Festenstein, Lacey, Skinner, Jenkins and Pepys, 1965). The hay harvested in rainy weather is, therefore, likely to be the most dangerous, and the incidence of the disease which is greater in the winter months depends upon the preceding harvest season and is highest in those parts with the greatest rainfall.

The serum of affected farmers contains precipitins against extracts of mouldy hay and especially against certain of the thermophilic actinomycetes present in great amounts in the mouldy hay. The most important of these is Micropolyspora $s p$. (Thermopolyspora polyspora). Micromonospora (Thermoactinomyces) vulgaris is also important but less so. The spores of Micropolyspora sp. are $1 \mu$ in size and can, therefore, penetrate into the alveoli where the tissue reactions of the disease occur. It has been calculated that a farmer working in a moderately dusty atmosphere due to mouldy hay will retain in his lungs 750,000 of these spores per minute (Lacey and Lacey, 1964).

The antigens in specimens of mouldy hay responsible for farmer's lung have been termed "farmer's lung hay" or FLH antigens. These are to be found in Micropolyspora sp. The sera of $87 \%$ of farmers with farmer's lung due to mouldy hay contain FLH precipitins and precipitins against other actinomycetes may be found in a further $2-3 \%$. In patients with farmer's lung due to other mouldy vegetable dusts, such as barley and oats, $50 \%$ have FLH precipitins. It is evident that there are a number of different sources of antigen which have not yet been found. Thus, the serological test may be negative in patients with all the features of farmer's lung and such a result does not exclude the diagnosis. On the other hand, 17 to $18 \%$ of exposed but unaffected farmers, or exposed farmers suffering from other lung diseases may give FLH reactions. A positive serological reaction does not, therefore, make the diagnosis by itself. It is necessary to take into account both clinical and immunological findings (Pepys and Jenkins, 1965).

The inhalation of extracts of mouldy hay in affected subjects provokes a febrile reaction coming on after 5 to 6 hours or so together with the other systemic and pulmonary changes typical of farmer's lung (Williams, 1963). Inhalation tests with extracts of Microplyspora sp. grown on ordinary culture mediac disease also provoke farmer's lung reactions in affecsubjects, thus confirming the role of this organism $\stackrel{5}{?}$ in the disease. A satisfactory skin test has not yeto been developed for farmer's lung.

Micropolyspora $s p$. is also important in a lung $\frac{\overline{\bar{s}}}{\bar{b}}$ disease in cattle, including in the fog-fever group. In® cattle suffering from fog-fever and exposed to mouldy hay $71 \%$ give FLH reactions on serological testing (Jenkins and Pepys, 1965).

The acute attacks of farmer's lung respond to $\vec{\omega}$ corticosteroid treatment but it is advisable to avoid further exposure wherever possible. Care in theo preparation and handling of hay and other cereal 3 . preparations is of value, to prevent sensitisation in the first place, and to prevent re-exposure to the thermophilic actinomycetes in the second.

The febrile episodes may suggest influenza-like ${ }^{\circ}$ illness or pulmonary infections due to a variety of $\infty$ different causes unless the association with exposure $\mathrm{O}$ to mouldy hay or other material is appreciated. The miliary infiltrations of the lungs may suggest sarcoidosis or miliary tuberculosis and the fibrotic $₫$ change may suggest diffuse interstitial fibrosis of 3 the type which follows fibrosing alveolitis. Th్ condition can be clearly distinguished from asthmo in which the reaction occurs in the bronchi and comes on rapidly with the production of wheezit dyspnoea. Only very few of the affected subjects have a previous history of asthma or of other allergie disorders.

Other diseases similar to farmer's lung are $\stackrel{0}{\circ}$ bagassosis due to the inhalation of the dust of $\stackrel{\mathbb{2}}{2}$ mouldy overheated sugar cane bagasse, used for $\overrightarrow{\overrightarrow{0}}$ the manufacture of insulation board, though the 3 specific causes in the bagasse have not yet been identified; and mushroom pickers' disease (Bringshurst, Byrne and Gershon-Cohen, 1959) in which 흘 Micropolyspora sp. is suspect.

\section{Bird Breeder's (Fancier's) Lung}

A farmer's lung type of disease has now been reported due to the inhalation of the dust of the excreta of pigeons and budgerigars (Reed, Sosman and Barbee, 1965; Hargreave, Pepys, Longbottom $D$ and Wraith, 1966). The bird fanciers, chiefly the pigeon fanciers, give a history of febrile reactions $N$ with malaise and loss of weight together with cough, dyspnoea and the appearance of crepitant rales, following some hours after cleaning out of $\omega$ pigeon-lofts. X-ray examination may show fine miliary infiltration of the lungs like that of farmer's lung. In the budgerigar fanciers however, the $\overparen{\Phi}$ disease tends to develop insidiously without recognisable acute febrile episodes, probably because of the smaller amount of antigen inhaled. The patients 
may present with advanced pulmonary fibrosis and with decrease in the $\mathrm{CO}$ transfer factor, and decrease in compliance of the lungs. In certain cases a bronchial asthmatic reaction to exposure may also be present, to be followed some hours later by a farmer's lung type of reaction affecting the peripheral gas-exchanging tissues.

On serological examination precipitins are present against the avian antigens which are to be found in extracts of the droppings of the birds and in their serum proteins. Intracutaneous tests with adequate concentrations of the extracts of the droppings or of the serum proteins give dual reactions like those mentioned above in the allergic form of pulmonary aspergillosis. These reactions consist of an immediate wealing reaction followed several hours later by large Arthus-type reactions. Inhalation tests with these extracts provoke a febrile response coming on 6 to 7 hours afterwards together with symptoms and signs of a reaction in the gas-exchanging tissues.

Avoidance of the birds may lead to considerable early improvement. Bird-breeder's lung should, therefore, be kept in mind for all patients with evidence of disease in the interstitial tissues of the lungs as shown by the clinical, physiological and radiographic findings already described. Bird breeder's lung is a hypersensitivity disease as shown by the recurrent attacks on exposure and the immunological findings. It should not be confused with virus diseases such as ornithosis or fungal disease such as cryptococcosis, which may be contracted from birds.

A similar picture to farmer's lung and bird breeder's lung may occur as a result of the inhalation of porcine and bovine pituitary snuff in the treatment of diabetes insipidus (Pepys, Jenkins, Lachmann and Mahon, 1965). In some of these patients asthma or disease of the interstitial tissues or both may develop. The X-ray may show fine miliary infiltrations of the farmer's lung type. Precipitins against porcine and bovine antigens are present in their sera, and skin tests with porcine and bovine serum proteins may give rise to dual, immediate and Arthus-type reactions. In addition to the differential diagnosis already listed for farmer's lung may be added eosinophil granuloma of the lungs, a disease associated with the causation of diabetes insipidus. In the patients affected by the pituitary snuff, however, the pulmonary manifestations develop after the onset of the diabetes insipidus and the other features of eosinophil granuloma such as invasion of bone have not been present.

\section{Conclusion}

These findings of recent years show that a strong immunological response is provoked by the inhalation of organic antigens so that further inhalations are responsible for different forms of lung disease, not only in the bronchi with the production of asthma, with or without pulmonary eosinophilia, but also in the peripheral gas-exchanging tissues leading to impairment of ventilationperfusion.

The sources of the responsible antigenic dusts are occupational, domestic and even therapeutic. It is important, therefore, to consider the possibility of hypersensitivity reactions to inhaled antigens in pulmonary disease of obscure etiology. It is very likely that additions will be made to the list of possible causes of such reactions now that the immunological mechanisms are better understood and can be applied to the identification of the sources of the reacting antigens.

\section{REFERENCES}

Bringshurst, L. S., Byrne, R. N., and GershonCOHEN, J. (1959): Respiratory Disease of Mushroom Workers, J. Amer. med. Ass., 171, 15.

DICKIE, H. A., and RANKIN, J. (1958): Farmer's Lung: an Acute Granulomatous Interstitial Pneumonitis Occurring in Agricultural Workers, J. Amer. med. Ass., 167, 1069.

Emanuel, D. A., Wenzel, F. J., Bowerman, C. I., and Lawton, B. R. (1964): Farmer's Lung. Clinical, Pathologic and Immunologic Study of Twenty Four Patients, Amer. J. Med., 37, 392.

Gell, P. G. H., and CoOMBS, R. R. A. (1963): Clinical Aspects of Immunology. Oxford: Blackwell.

Festenstein, G. N., Lacey, J., Skinner, F. A., Jenkins, P. A., and PEPYS, J. (1965): Self-heating of Hay and Grain in Dewar Flasks and the Development of Farmer's Lung Antigens, J. gen. Microbiol., 41, 389.

Hargreave, F. E., PePys, J., Longbottom, J. L., and WraITH, D. G. (1966): Bird Breeders' (Fanciers') Lung, Lancet. (In press).

Jenkins, P. A., and PePYS, J. (1965): Fog-fever. Precipitin (F.L.H.) Reactions to Mouldy Hay, Vet. Rec., 77, 464.

LACEY, J., and LACEY, M. E. (1964): Spore Concentrations in the Air of Farm Buildings, Trans. Brit. Mycol. Soc., 47, 547.

Longbottom, J. L., and PePYS, J. (1964): Pulmonary Aspergillosis: Diagnostic and Immunological Significance of Antigens and C-Substance in Aspergillus fumigatus, J. Path. Bact., 88, 141.

Pepys, J., Riddell, R. W., Citron, K. M., Clayton, Y. M., and SHORT, E. I. (1959): Clinical and Immunologic Significance of Aspergillus fumigatus in the Sputum, Amer. Rev. resp. Dis., 88, 167.

PePYS, J., and JenKINS, P. A. (1965): Precipitin (F.L.H.) Test in Farmer's Eung, Thorax, 20, 21.

Pepys, J., Jenkins, P. A., LachmanN, P. J., and Mahon, W. E. (1965): An Iatrogenic Auto-antibody: Immunological Responses to Pituitary Snuff in Patients with Diabetes Insipidus, J. Endocr. (Proceedings of the Soc. for Endocrinol.), 33, viii (and in print).

Reed, C. E., Sosman, A., and Barbee, R. A. (1965): Pigeon Breeders' Lung. A Newly Observed Interstitial Pulmonary Disease, J. Amer. med. Ass., 193, 261.

WILLIAMS, J. V. (1963): Inhalation of Skin Tests with Extracts of Hay and Fungi in Patients with Farmer's Lung, Thorax, 18, 182. 